\title{
COORDENAÇÃO, AVALIAÇÃO E ACOMPANHAMENTO DOS PROJETOS DE PESQUISA EM SAUDE DO PROGRAMA POLONOROESTE
}

\author{
Waldmir BELINATI (1) \& Aluizio COSTA e SILVA (2)
}

\begin{abstract}
RESUMO
As atividades de Coordenação, Acompanhamento e Avaliação do Programa de Desenvolvimento Integrado do Noroeste do Brasil - POLONOROESTE - são analisadas, com base nos objetivos gerais e específicos do Programa e nos resultados parciais alcançados pelos pesquisadores, até o presente momento. Destaque especial é dado à auditagem técnica dos projetos de pesquisa, realizada pela atual Coordenação de Ciências da Saúde, que passou a incrementar, a partir de 1986, as atividades de assessoramento e acompanhamento continuados dos projetos, prestação de serviços de consultoria e início de um processo de treinamento de recursos humanos locais envolvidos nos projetos em centros mais avançados. Ao final de cinco anos de existência do Programa, os resultados alcançados são considerados positivos e os benefícios dos mesmos inquestionáveis, principalmente quando se sabe que os problemas de saúde da região alvo do POLONOROESTE, até o início do Programa, eram pouco conhecidos, em função da inexistência de estudos sobre as condições de saúde-doença da população e de dados que permitissem orientar a implementação de uma política de saúde adequada e prioritária para a Região. Os resultados dos projetos sobre história natural da malária, mosquitos vetores, imunopatologia da doença e estudos visando ao processo saúde-doença são analisados, concluindo-se que os mesmos darão suporte à planificação das futuras açōes de saúde e à aplicação de soluções adequadas às necessidades da população da área de abrangência do POLONOROESTE.
\end{abstract}

UNITERMOS: Avaliação de projetos de pesquisa; Projetos de pesquisa em saúde; Polonoroeste.

\section{INTRODUÇÃO}

O presente fascículo da Revista do Instituto de Medicina Tropical de São Paulo objetiva levar ao conhecimento da comunidade científica informações referentes ao financiamento, desenvolvimento e resultados dos projetos de pesquisa em saúde do Programa de Desenvolvi- mento Integrado da Região Noroeste do Brasil - Polonoroeste.

Os projetos de pesquisa, que estão a cargo de diversas instituições, financiadas através do CNPq, requerem a existência de um sistema complexo de coleta de informações, para ava-

(1) Médico, Analista de Desenvolvimento Científico da Coordenação de Ciências da Saúde do Conselho Nacional de Desenvolvimento Cientifico e Tecnológico, Brasilia, DF, Brasil.

(2) Médico, Coordenador de Ciências da Saúde do Conselho Nacional de Desenvolvimento Científico e Tecnológico, Brasília, DF, Brasil.

Endereço para correspondência: Dr. Waldmir Belinati. Conselho Nacional de Desenvolvimento Científico e Tecnológico CNPq. Av. W-3 Norte, Quadra 507, Bloco B. Caixa Postal 11.1142. CEP 70740 Brasilia, DF, Brasil. 
BELINATI, W. \& COSTA E SILVA, A. - Coordenação, avaliação e acompanhamento dos projetos de pesquisa em saúde do Programa Polonoroeste. Rev. Inst. Med. trop. Sāo Paulo, 30(3):125-136, P988.

liação do andamento de cada um dos projetos e do impacto do Programa na população. O andamento dos projetos tem sido avaliado em função das metas físico-financeiras propostas para cada período, utilizando-se principalmente dos dados obtidos através dos relatórios técnicos e financeiros e de observações recolhidas em campo. O impacto na população é medido, servindo-se de informações oficiais sobre a evolução das condições de saúde e dos resultados obtidos nos projetos de pesquisa realizados na Região, tanto de morbi-mortalidade quanto do acesso aos serviços de saúde.

O presente fascículo compreende uma análise da situação atual do Programa, em face dos seus objetivos iniciais, contemplando os aspectos relativos ao desenvolvimento dos projetos na área de abrangência do Polonoroeste e as pesquisas operacionais visando ao combate da malária. Uma análise econômica das operações de controle da malária e das necessidades em saúde da Região está sendo realizada com dados obtidos a partir dos resultados parciais alcançados com o Programa, e será objeto de outra publicação. Limitar-nos-emos, por ora, a apresentar os objetivos e dados históricos de avaliação e acompanhamento do Programa, com o produto dos projetos em andamento: os resultados parciais alcançados pelos pesquisadores até o momento, razão maior desta publicação.

\section{Breve Esboço Histórico e Objetivos do Polonoroeste}

O Programa de Desenvolvimento Integrado do Noroeste do Brasil (Polonoroeste) nasceu com os objetivos gerais de pavimentar a estrada que une Cuiabá a Porto Velho (BR-364), área de corıiderável potencial de produção, objeto de rápidos fluxos migratórios, e, ao mesmo tempo, proporcionar os investimentos necessários ao desenvolvimento sócio-econômico ordenado da área influenciada pela estrada e à proteção do ambiente físico e da população indígena da Região.

A pavimentação da BR-364 visou ao aumento da contribuição, à economia nacional, dos recursos físicos subutilizados da Região, à redução dos custos dos transportes e melhora dos termos do comércio entre a Região e as áreas mais desenvolvidas e populosas situadas a) Sul.
Contudo, a melhoria da BR-364 tornou sua área de influência mais acessivel a migrantes em potencial, exercendo maior pressão sobre o ambiente físico e social da área. Dessa maneira, para ajudar a evitar o desenvolvimento desordenado, que poderá ameaçar irreversivelmente os próprios recursos que a pavimentação da BR-364 contribuirá para desenvolver, estão sendo estudadas políticas e instituídas estruturas para suas aplicações. Sem um adequado ordenamento, a contínua migração resultará, a médio e longo prazos, em prejuízos ao ambiente e à sociedade, decorrentes da ocupação de terras mais pobres, sujeitas a práticas agrícolas carentes de adaptação, o desperdício de valiosas madeiras, a invasão de reservas indígenas e naturais, a concentração da propriedade da terra num grupo relativamente pequeno de titulares e aglomeração de migrantes em áreas urbanas sem condições de recebê-los.

O Decreto Presidencial, de 27 de maio de 1981, definiu os objetivos e recursos do Programa, que se basearam em amplos estudos realizados anteriormente na respectiva área. Consoante o Decreto, os objetivos gerais do Polonoroeste são os seguintes:

a) Integrar as regiões marginais da economia nacional;

b) Assegurar o povoamento ordenado;

c) Aumentar a produtividade, renda, saúde e bem-estar social dos migrantes;

d) Proteger as comunidades indígenas, os recursos da terra e do ambiente.

\section{Estimativa do Custo do Programa}

O custo total do Polonoroeste foi estimado, preliminarmente, em US $\$ 1,5$ bilhões. Destes, cerca de $53 \%$ foram destinados à construção de estradas, $40 \%$ ao desenvolvimento rural e assentamentos, e o saldo para saúde, proteção aos índios, ao ambiente e para administração.

Os fundos foram aprovados pelo Ministério do Planejamento, em coordenação com a Superintendência de Desenvolvimento do Centro-Oeste - SUDECO, do Ministério do Interior, que coordena e administra todas as atividades do Programa.

\section{A Área do Programa Polonoroeste}

Com uma área aproximada de 410.000 $\mathrm{km}^{2}$, a Região Noroeste do Brasil equivale a 
BELINAT!, W. \& COST A E SILVA, A. - Coordenação, avaliação e alompanhamento dos projetos de pesquisa em saúde do Programa Polonorveste. Kev. Inst. Med. trop. São Paulo, 30(3): 125-136, 1988.

cerca de três quartos da superfície da França, embora corresponda a pouco menos de $5 \%$ do território do Brasil. A Região inclui todo o Estado de Rondônia e 14 municípios do Oeste do Estado de Mato Grosso, todos oficialmente considerados incluídos na área de influência da rodovia BR-364, entre Cuiabá e Porto Velho. Em 1980, a população regional era estimada em 1.000 .000 habitantes, com um crescimento de $10,8 \%$ ao ano durante a última década $(7,8 \%$ ao ano em Mato Grosso e $15,8 \%$ em Rondônia). A densidade média da Região ainda é inferior a três habitantes por $\mathrm{km}^{2}$, e sua maior concentração ocorre nas localidades e colônias agrícolas instaladas ao longo da BR-364. O desenvolvimento sócio-econômico de Rondônia, igualmente, concentra-se quase exclusivamente ao longo do eixo a citada rodovia.

\section{O Projeto de Saúde do Polonoroeste}

\section{- Algumas caracteristicas do setor saúde, pro- blemas e questões}

A população do Brasil, de mais de $135 \mathrm{mi}-$ lhões de habitantes, está desigualmente distribuída por sua área de 8,5 milhōes de $\mathrm{km}^{2}$. As densidades populacionais variam largamente, entre menos de 2 habitantes por $\mathrm{km}^{2}$ na Bacia Amazônica até 60 habitantes por $\mathrm{km}^{2}$ no Sudeste. Esta população tão desigualmente distribuída deve-se à dificuldade de acesso a regiões inteiras (Amazônia), a um padrão histórico de colonização baseado no desenvolvimento das colheitas (Nordeste) e mais recentemente à indústria (Sudeste). Nos últimos anos tem havido uma contínua e grande migração das grandes e pobres áreas rurais do Nordeste para os polos industriais do Sul e, ainda mais recentemente, do Sul e Sudeste para as áreas rurais promissoras e inexploradas da Amazônia Norte e Nordeste.

Este padrão migratório provoca problemas de saúde resultantes do baixo nivel de vida e nutrição, faz surgir favelas à margem das grandes cidades, dificultando aos serviços de saúde a cobertura das necessidades desta população, especialmente na área da Amazônia, expõe grande número de migrantes sem imunidade às condições ecológicas que favorecem a propagação de doenças, particularmente a malária.

Os mais importantes fatores que influen- ciam a saúde dos habitantes de Rondônia tornam os serviços de saúde custosos e difíceis. São eles: população rapidamente crescente e dispersa; falta de infra-estrutura de transporte e comunicações; difícil acesso aos serviços essenciais; condições climáticas favoráveis à propagação de doenças endêmicas; educação precária e a insuficiente cobertura dos serviços de saúde, cujas unidades de serviços estão localizadas, principalmente, ao longo da BR-364 e virtualmente nào existem no interior das áreas colonizadas. A resultante alta morbidade e alta mortalidade afetam adversamente o bem comum e a produtividade de uma área de desenvolvimento agricola e pecuária, das mais atraentes do País.

As condições gerais de saúde em Rondônia e Mato Grosso são precárias, comparadas ao restante do País. A taxa de mortalidade infantil é de 128 por mil nascidos vivos. As complicacões mais comuns são as perinatais e as infecções intestinais, malária, pneumonia e sarampo. Estas, juntamente com as doenças do trato respiratório, acidentes e doenças cerebrovasculares são as maiores causas de mortalidade para todas as idades.

A malária é o mais importante problema de saúde de Rondônia. As extensas florestas de Rondônia, o alto acúmulo de águas de chuva na superficie, a umidade e a alta temperatura formam o habitat ideal do A. darlingi, mosquito portador da malária. A população rapidamente crescente, o difícil acesso a muitos povoados e fazendas, o inadequado serviço de saúde e, principalmente, a falta de recursos financeiros, tornam o controle da malária extremamente difícil.

As estatísticas oficiais mostram a seriedade da situação. Em 1980, mais de 62.000 casos de malária, resultando numa perdá de cerca de um milhào de dias de trabalho, foram identificados em Rondônia. Estes compreendiam mais de um terço dos 170.000 casos identificados em um total de população nacional de 119 milhões de habitantes, para a época. Devido à falta de informações, este quadro com certeza não mostra exatamente a gravidade da situação em Rondônia. Pode-se assegurar que toda familia residente em Rondônia hoje já teve pelo menos um de seus membros afetados pela malária. Cerca de $40 \%$ de todos os casos correm risco de vida pela infecção pelo Plasmodium falciparum. Além do mais, a resistência à droga anti-malárica, a cloroquina, está aumentando. Em vista de mui- 
BELNATI, W \& COSTA E SILVA, A. - Coordenação, avalią̧ão e acompanhamento dos projetos de pesquisa ern saude do Programa Polo noweste. Rev Inst. Med, irop. Säo Paulo, 30(3):125136, 1988

tos colonizadores virem de areas isentas de malarıa e, portanto, sem nenhum imunização, na ausencia de tratamento completo e adequado, muitos morrem desta doença.

Outras entidades contribuem para o agravamento do quadro de saúde de Rondônia. A falta de suprimento de água e de sistema de esgoto adequados, bem como as instalaçōes provisórias no meio rural fazem das infecçoes gastrointestinais e respiratórias as mais propagadas depois da malária. Juntas, elas compreendem $75 \%$ de aproximadamente 180.000 casos iratados nos centros de saúde em 1980, e são as principais contribuintes para as altas taxas de morbi-mortalidade em adultos e criancas. Vestes casos tambèm é dificil encontrar-se uma familia rural de Rondônia que não tenha sido afetada por essas e outras doenças, tais como sarampo, diarréia e hepatite infecciosa.

De 1980 a 1983 , indicadores sugeriam uma melhora contínua da situação da malária: a incidência anual do parasita diminuiu de 120 para 87, registrando-se. entretanto, elevações contínuas, de 105 , ao final de 1983 , para 127 , em meados de 1984, e 160, ao final de 1986 (dados referem-se ao IPA - indice parasitário anual, correspondendo ao número de casos de malária por 1000 habitantes). Apenas $41 \%$ das casas programadas receberam dedetização no primeiro semestre de 1985 , comparados com $90 \%$ no primeiro semestre de 1979.

Alguns dados epidemiológicos da malária em Rondônia, em 1980 e 1986, merecem ser destacados:

Em 1980, o Estado contava com uma população de 493.000 habitantes. Neste ano, foram examinados 180.149 lâminas pela SUCAM, registrando-se 59.178 positivas, sendo 26.269 para o P. falciparum. O índice parasitário anual foi de 120 por 1.000 habitantes.

Em 1986, para uma população de 1.205.000 habitantes no Estado, foram examinadas 561.355 lâminas, registrando-se 191.727 positivas, sendo 110.142 para o $\mathbf{P}$. falciparum. $O$ indice parasitário anual foi de 160 por 1000 habitantes.

Os fatores principais do agravamento da situação da malária seriam atribuidos:

a) A migraçào humana incontrolada de susceptiveis em Rondônia;

b) Ao crescimento da densidade das populacões de mosquitos, devido à expansão da area alagada emi novas frentes de colonização agrícola;

c) A maior exposiçăo interna, pela ausència de proteção (corno borrificaçào das paredes com DDT, larvas de mosquiros, etc) e ao aumento da expusiçào externa, especialmente durante atividades na agricultura e no garimpo;

d) A existência de um reservatório aproppriado da malária, consequêencia do alto número de casus de doençá tratados a à resistencia do parasita (35\%) ao tratamento; e

e) A problemas de gerenciamento operacional de firmeza de propósitos nas atividades de controle da malária.

\section{- Sub-programa de pesquisa em saúde do Polonoroeste}

O contrato firmado entre o Governo Brasileiro e o Banco Internacional de Reconstrução e Desenvolvimento (BIRD) prevê o fortalecimento da capacidade de pesquisa em saúde das instituições regionais, a caracterização dos problemas de saúde (do ponto de vista epidemiológico) e o estudo para o controle e tratamento de doenças endêmicas e outras com risco de endemização na área.

O Conselho Nacional de Desenvolvimento Científico e Tecnológico $(\mathrm{CNPq})$, visando à preparação de um programa integrado de pesquisas, elaborou, com a participação de cientistas brasileiros, uma proposta inicial com três segmentos principais:

1. Estudo das doenças endêmicas locais;

2. Avaliação dos riscos dessas endemias para as populações afluentes;

3. Avaliação do risco de implantação de novas endemias na área de influência da BR-364 (Cuiabá-Porto Velho).

O processo de discussão que se seguiu à elaboração da proposta inicial mostrou a necessidade de uma abordagem mais ampla e profunda dos aspectos das condições de saúde da Região, na qual fosse possivel traçar um perfil inicial dessas condições. Nesse sentido, o envolvimento das instituições prestadoras de serviços de saúde - Secretaria de Estado de Saúde de Rondônia - SES, RO, Secretaria de Estado de Saúde de Mato Grosso - SES, MT, Superintendência de Campanhas de Saúde Pública - 
BIL L INATI, W \& COSTA E SIL VA, A. - Coordenaçào, avaliação e acompanhamento dos projetos de pesquisa em saúde do Programa Polonoroeste. Rev. Inst. Med. trop. São Paulo, 30(3):125-136, 1988

SUCAM, Fundação Serviços de Saúde Pública - SESP, entre outras, tornaram-se elementos intermediários para a consecução dos objetivos da proposta.

\section{- Objetivo geral do sub-projeto de pesquisa em saúde}

Os objetivos principais do componente Pesquisa em Saúde do Polonoroeste, como originariamente concebido, são:

1. Apoio às ações dos serviços de saúde na área;

2. Criação de infra-estrutura permanente de investigação científica de saúde na Regiâo, fortalecendo, consequientemente, os núcleos locais de Pesquisa em Saúde.

\section{- Objetivos Específicos do Sub-Projeto de Pesquisa em Saúde}

1. Realizar diagnóstico de saúde da área do Polonoroeste, voltado para a caracterização epidemiológica da mortalidade, morbidade, prevalência de doenças infecciosas, situação nutricional da população e suas implicações sócio-econômicas e ecológicas;

2. Estabelecer um sistema de vigilância epidemiológica para acompanhar as transformações ocorridas na saúde da população, a partir da implantação do Programa;

3. Propor, com base no diagnóstico, metas prioritárias para a implantação de ações de saúde que venham a intervir sobre o processo saúde/doença na região;

4. Realizar pesquisas operacionais em modelo de prestação de serviços de saúde;

5. Caracterizar a endemia malárica na área, no que diz respeito a sua prevalência e periodicidade estacional, vetores de importância epidemiológica, papel da migração humana e das condições habitacionais na dinâmica de transmissão da doença;

6. Testar a sensibilidade do Plasmodium "in vitro" e "in vivo" a drogas novas e tradicionais;

7. Estudar métodos não convencionais para controle da malária, abordando características da população humana e dos vetores;

8. Estudar o vetor da malária quanto à sua biologia e ecologia;

9. Estudar os agentes etiológicos, vetores e re- servatórios que compõem a cadeia de transmissão da leishmaniose tegumentar;

10. Comparar o comportamento das variáveis epidemiológicas com taxas de incidência e prevalência em pessoas resistentes na região da BR-364;

11. Avaliar o estado imunitário dos imigrantes e dos nativos em relação aos dois tipos mais comuns de hepatite.

\section{- Descrição Geral dos Objetivos do Sub-Programa de Pesquisa em Saúde do Polonoroeste}

O Programa Polonoroeste, componente de Pesquisa em Saúde, desde sua implementação, manifestou, com clareza, com um de seus objetivos básicos "o fortalecimento da capacidade de pesquisa em saúde, incluindo pesquisas nas áreas de controle/tratamento da malária e leishmaniose, considerando-se que a malária é o mais importante problema de saúde em Rondônia e que muito pouca pesquisa tem sido realizada por falta de fundos, a despeito das pesquisas operacionais sobre o comportamento do mosquito, drogas anti-maláricas e estudos epidemiológicos para auxiliar as operaçôes de controle' 1.2

O conjunto de pesquisas em malária, conforme inicialmente proposto, consiste de três grupos:

\section{Entomologia}

Do ponto de vista entomológico, o projeto visou a:

a) Estudar o comportamento do transmissor mais importante conhecido, o Anopheles darlingi, após as profundas alterações ecológicas conseqüentes à abertura de grandes estradas e intensa migração para atividades agropecuárias e de mineração;

b) Procurar outros eventuais transmissores entre espécies de anofelinos, até o presente considerados como sem importância na veiculação da malária;

c) Estudar o comportamento dos transmissores quanto à sua variação sazonal, endofilia, antropofilia, frequência horária de hematofagismo, distribuição vertical na mata e criadouros;

d) Estudar a possibilidade de que os mosquitos 
BELINATI, W. \& COSTA E SILVA. A. - Coordenaçāo, avaliação e acompanhamento dos projetos de pesquisa em saúde do Programa Polonoroeste. Rev. Ins1. Med. Irop. São Paulo, 30(3):125-136, 1988.

atribuidos a uma mesma espécie de hábitos diferentes, em áreas distintas, constituam complexos de espécies crípticas;

e) Efetuar o acompanhamento entomológico de medidas de controle efetuadas na cidade de Ariquemes, pela SUCAM e Secretaria de Saúde de Rondônia.

\section{Imunologia}

O grupo de estudos de imunologia da malária teve como objetivo comum contribuir para a introdução e desenvolvimento de métodos imunológicos necessários ao controle da malária.

Foram efetuados estudos transversais e longitudinais, sendo que os primeiros visando a avaliar a aquisição de imunidade protetora pela população migrante e as repercussões imunopatológicas relacionadas à malária. Através de estudos longitudinais, estabeleceu-se o acompanhamento da resposa imune do individuo submetido a infecções repetidas pelo Plasmodium.

Esperava-se que os resultados obtidos contribuissem para os seguintes objetivos finais:

1. Colaborar para o desenvolvimento de estudos imunopatológicos;

2. Colaborar para a elaboração mais racional de projetos de colonização destinados à Amazônia;

3. Fornecer subsidios para o futuro emprego das vacinas anti-maláricas.

O elenco de projetos iniciais previa a implantação de uma rede de laboratórios soroepidemiológicos destinada a estabelecer o registro adequado das condições imunitárias de populações selecionadas. Este estudo servirá de base à avaliação da eficácia e efetividade de medidas anti-maláricas e, inclusive, no futuro, de testes para avaliação de vacinas. Essa rede de laboratórios, além de desenvolver pesquisas imunológicas básicas, poderá garantir o estudo epidemiológico continuado de populações selecionadas, de acordo com as finalidades específicas de cada projeto e tendo como objetivo o controle melhor da doença.

Os laboratórios de execucão dos levantamentos poderào obter amostras de sangue de pacientes infectados, de modo a fornecer antigenos representativos da população enferma. Os laboratórios que atualmente realizam cultura de $\mathbf{P}$. falciparum estarão em condições de fornecer antígenos para referência e uso de rotina nos testes.

\section{Clínico-Terapêutico}

As pesquisas neste grupo visaram basicamente ao estudo da resistência do plasmódio a drogas, tanto "in vitro" quanto "in vivo", além de avaliar clinica e laboratorialmente a di. nâmica e a natureza de aquisição da imunidade à malária falciparum em migrantes.

Todos os grupos, cujos projetos foram anteriormente enfocados, atuam no sentido de realizar uma abordagem epidemiológica para estabelecer as características da malária na Região.

\section{Coordenação, Acompanhamento e Avaliação do Polonoroeste}

A Coordenação, Acompanhamento e Avaliação do Componente Ciência e Tecnologia Pesquisa em Saúde do Programa Polonoroeste exigiu amplo esforço de articulação interinstitucional e integração entre os diversos subcomponentes de pesquisa, para alcançar-se os objetivos gerais e especificos do Programa como um todo.

A diversidade de sub-setores do conhecimento técnico-científico atuantes nas pesquisas em saúde, a necessidade de harmonizaçâo dos objetivos especificos de cada sub-componente e a atividade de gerenciamento das pesquisas justificam o elemento Coordenação, Acompanhamento e Avaliação.

O contrato de empréstimo 2061 BR - Programa de Desenvolvimento da Região Noroeste - Primeira Fase - Projeto Saúde, firmado em 15 de dezembro de 1981 , entre a República Federativa do Brasil e o Banco Internacional para Reconstrução e Desenvolvimento - BIRD atribuiu ao $\mathrm{CNPq}$, como órgão coordenador da pesquisa científica e tecnológica no Brasil, a execução do Projeto de Pesquisa em Saúde, acompanhamento e avaliação, cujas metas seriam atingidas através de reforço para a pesquisa e promoção à institucionalização, ficando o CNPq comprometido a firmar contratos com as instituiçôes de pesquisas locais, para pesquisar malária e outras doenças prevalentes.

A atuação da Coordenação de Saúde e $\mathrm{Nu}$ trição, a partir de maio de 1983, teve como maior objetivo implantar na região alvo do programa uma infra-estrutura de pesquisas e recur- 
BELINATL, W. \& COSTA E SILVA, A, - Coordenação, avaliação e acompanhantento dos projetos de pesquisa em saude do Programa Potonoroeste. Rev. Inst. Med. trop. São Paulo, 30(3):125-136, 1948

sos humanos capaz de consolidar-se e propor atividades de investigação adequados à solução de problemas de saúde regionais.

A variedade de problemas de saúde apresentados pela região de implantação dos projetos de colonização da rodovia BR 364 gerou, logo no início das atividades do componente de pesquisas em saúde, uma grande expectativa da comunidade científica nacional. Esta visão dos pesquisadores acarretou, em consequêencia, uma demanda ao programa de vários tipos de projetos voltados para a Região, embora nem todos tivessem sintonia com os objetivos propostos, e nem sempre levassem em conta as prioridades regionais em suas elaborações.

Tal situação foi objeto de reuniões com membros de instituições de pesquisa, que apresentaram anteprojetos visando à coordenação inicial das pesquisas, estruturados a princípio de modo isolado. Assim, a antiga Coordenação de Saúde e Nutrição (CSN) partiu de um envolvimento inicial com 8 projetos de pesquisa, apresentados por cinco instituições, para um número de 6 projetos, conduzidos por 4 instituiçoes executoras. Estes projetos foram objeto de fomento e acompanhamento pela CSN e posteriormente pela Coordenação de Ciências da Saúde (a partir de 1986). Devido à carência de recursos que permitissem um diagnóstico das condições de saúde da região alvo, fez-se necessário que os investimentos nos projetos de diagnóstico fossem inicialmente privilegiados.

Manteve-se aberto espaço à pesquisa básica em vetores, agentes causais e hospedeiros intermediários de malária e leishmaniose, inicialmente, respeitadas as dimensões das necessidades de investimentos das pesquisas em serviços de saúde. Para o exercicio 1985/86, além dos projetos de pesquisa iniciados a partir de 1984 , três novos projetos da Universidade Federal de Mato Grosso do Sul foram aprovados e passaram a ser financiados através do CNPq. A Tabela 1 consolida as informaçôes administrativas e financeiras de todos os projetos financiados, de 1982 até o presente momento.

Embora haja dificuldades de fixação de quadros técnicos na área do Polonoroeste, principalmente em Rondônia, em decorrência de inexistir na Região infraestrutura para investigação científica, o CNPq patrocinou, de 1984 a 1987, a ida à Região (Rondôniá e Mato Grosso) de consultores da mais alta qualificação no País, em campos específicos (metodologia de pesquisa, epidemiologia, bioestatística, nutricão e informática, dentre outros) para auxiliar os pesquisadores locais na elaboração e desenvolvimento de seus projetos de pesquisa. Apesar deste esforço por parte do CNPq, os projetos, como um todo sofreram atrasos significativos nos seus cronogramas, em função tanto das dificuldades para estruturação das equipes de pesquisadores locais, como dos resultados de entraves administrativos na liberação dos recursos aos pesquisadores.

Apesar das dificuldades e questionamentos citados, o saldo, ao final de cinco anos do Programa, é extremamente positivo e os benefícios do mesmo inquestionáveis, principalmente quando se sabe que os problemas da região alvo do POLONOROESTE, até o início do Programa, eram pouco conhecidos, em função da inexistência de estudos sistemáticos sobre as condições de saúde-doença da população e de dados que permitissem orientar a implementação de uma política de pesquisa em saúde adequada e prioritária para a Região. Os resultados dos projetos sobre a história natural da malária, mosquitos vetores, imunopatologia da doença, estudos visando ao processo saúde-doença, principalmente de inquéritos epidemiológicos e nutricionais realizados pelos pesquisadores locais, além de terem contribuido para o início de um processo de consolidação do núcleo de pesquisa em saude da Universidade Federal de Mato Grosso, são muito relevantes e fornecerão subsídios para o planejamento e programação das ações de saúde na área de abrangência do POLONOROESTE.

\section{CONCLUSÃO}

A atual Coordenação de Ciências da Saúde do $\mathrm{CNPq}$, desde 1986, vem realizando uma auditagem técnica de todos projetos de pesquisa, incrementand $\rho$ as atividades de assessoramento técnico e de acompanhamento continuados dos projetos, através de supervisão dos mesmos "in loco", prestação de serviços de consultoria e início de um processo de treinamento de recursos humanos locais envolvidos nos projetos em centros mais avançados.

Para a consecução dos objetivos citados, a nova Coordenação constituiu uma Comissão permanente para avaliar e redirecionar as atividades do sub-componente de pesquisa em saúde 


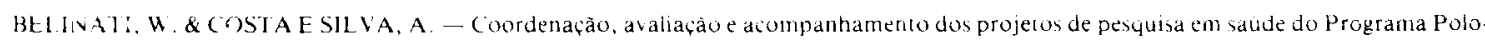
noroestc. Rev. Inst. Med, trop. Sào Paulo, 30(3):125.136, 1988

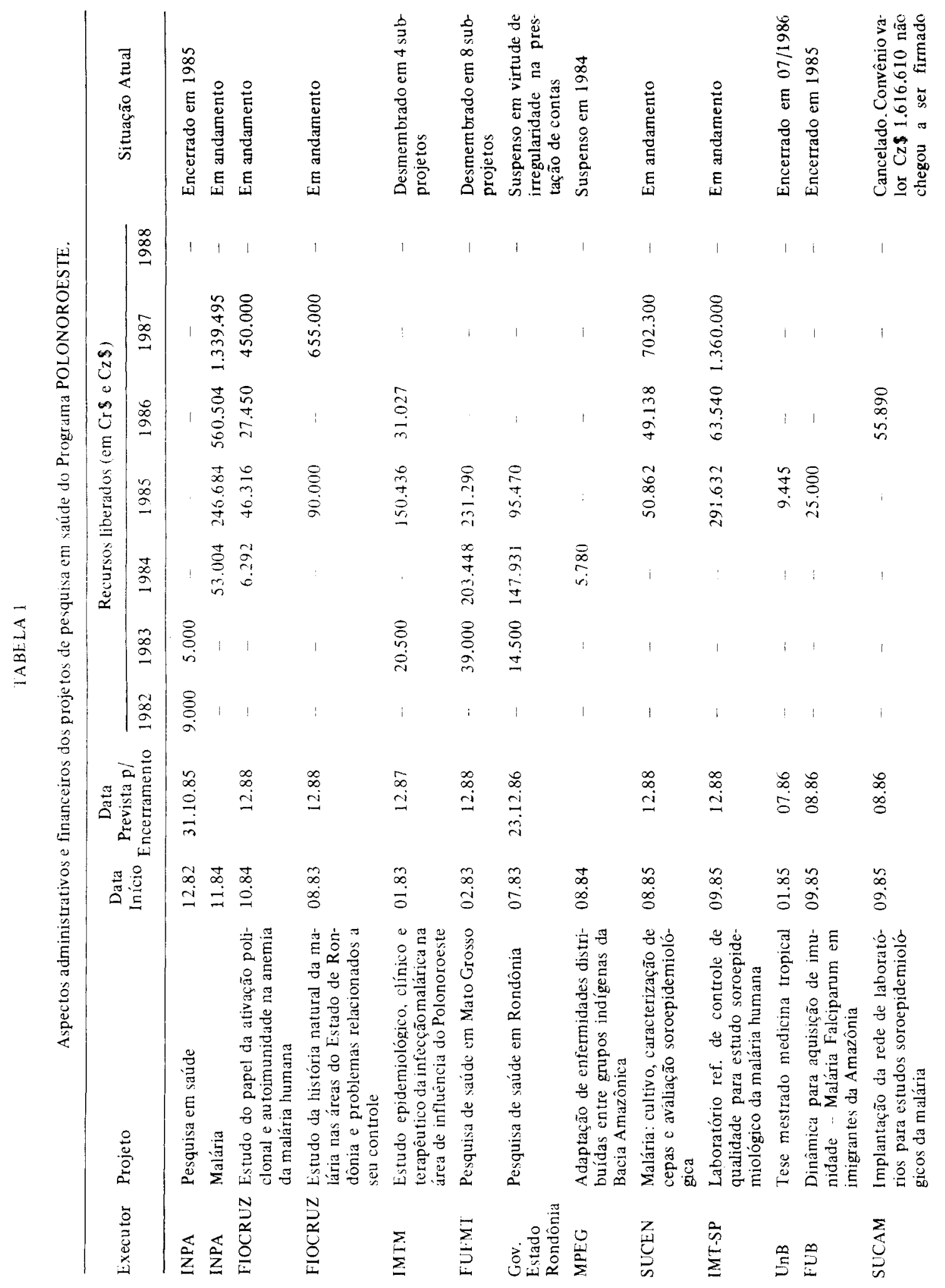


BELINATI, W. \& COSTA E SILVA, A. - Coordenaça, avaliaçào e acompanhamento dos projetos de pesquisa em saúde do Programa Poionoroeste. Rev. Inst. Med. trop. São Paulo, 30(3):125-136, 1988.

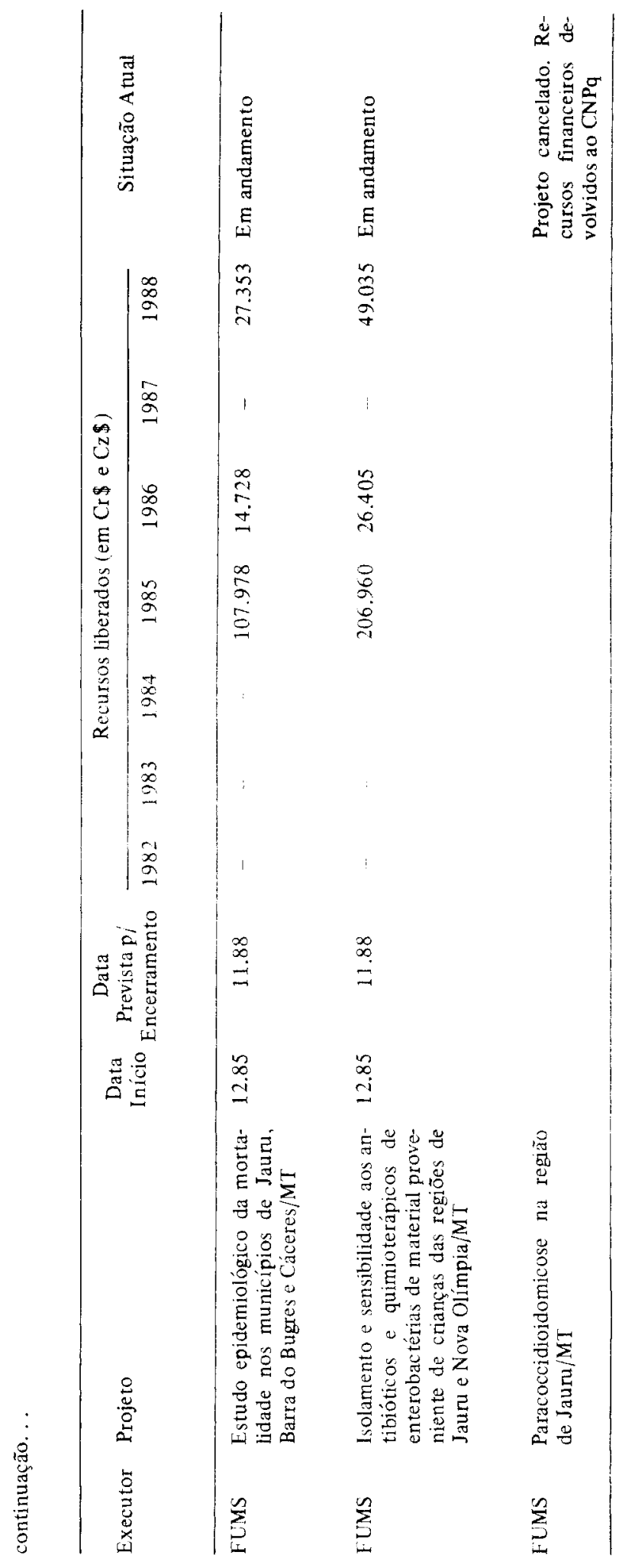


BELINATI, W \& COSTA E SILVA, A. - Coordenação, avaliaçăo e acompanhamento dos projetos de pesquisa em saúde do Programa Polonoroeste. Rev. Inst. Med. trop. São Paulo, 30(3):125-136, 1988.

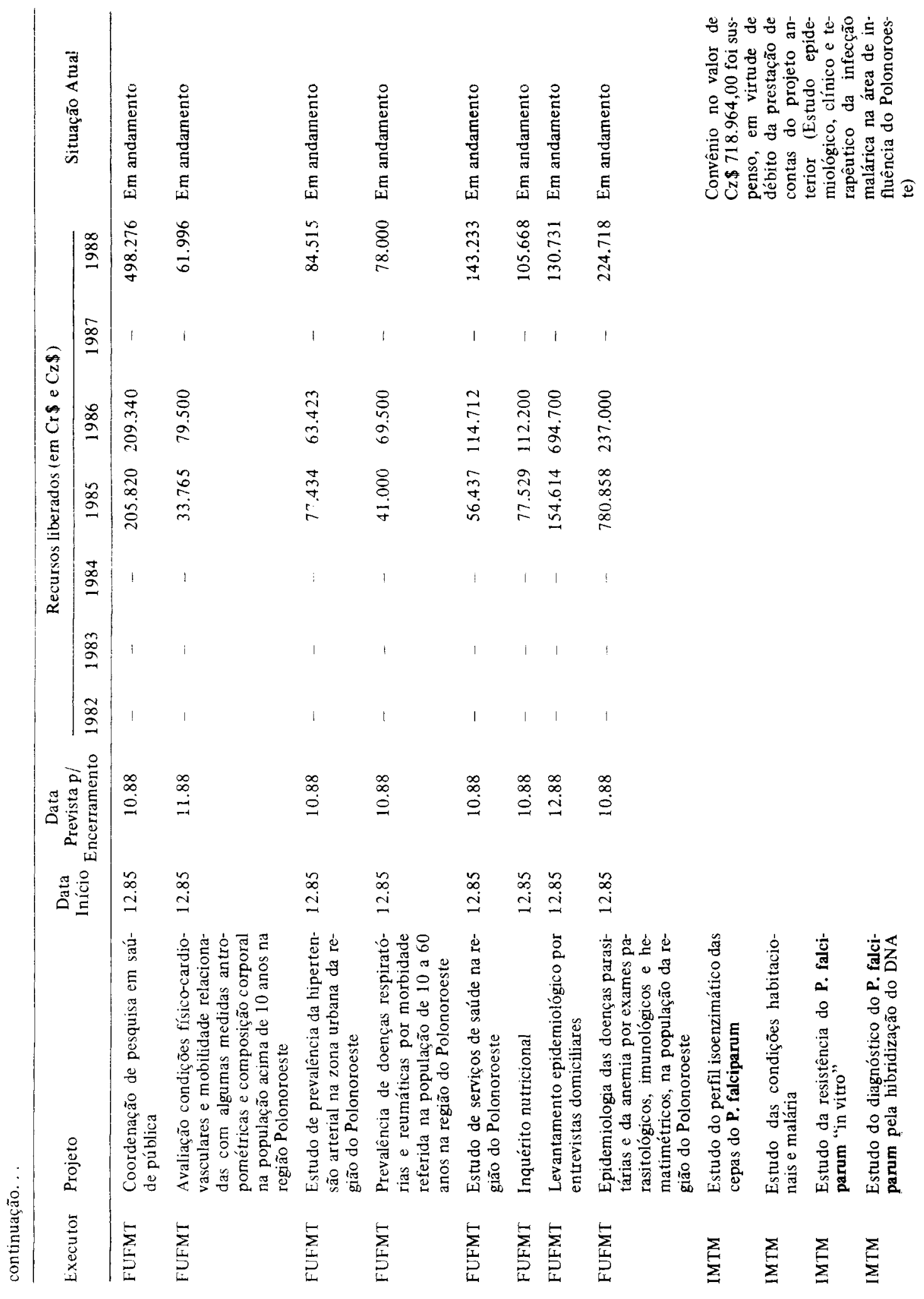


BELINATI, W. \& COSTA E SILVA, A. - Coordenação, avaliação e acompanhamento dos projetos de pesquisa em saúde do Programa Polonoroeste. Rev. Inst. Med. trop. Sāo Paulo, 30(3):125-136, 1988.

do Polonoroeste e obter informações objetivas quanto a metas, recursos, gerenciamento e aspectos qualitativos de cada um dos projetos de pesquisa. Da comissão participaram os Doutores Mário Rubens Montenegro (Coordenador), Henrique Lenzi, José Brandão Neto, Aluízio da Costa e Silva, Aguinaldo Gonçalves e Waldmir Belinati.

$\mathrm{Na}$ análise e reavaliação dos projetos de pesquisa em saúde efetuadas, procurou-se adequar os mesmos às reais necessidades da $\mathrm{Re}_{-}$ gião, contemplando, prioritariamente, a capacitação de recursos humanos locais para o desenvolvimento dos projetos.

Apesar de muitos percalços enfrentados, os avanços ocorridos durante a atual gestão do componente pesquisa em saúde do Polonoroeste refletem-se no esforço considerável desenvolvido pelos técnicos e pesquisadores do Programa para divulgar o produto final de suas pesquisas. Dezenas de trabalhos já foram publicados em revistas de prestígio nacional e internacional, constituindo-se o presente fascículo da Revista do Instituto de Medicina Tropical no exemplo maior da capacidade de realização dos pesquisadores que participam desta edição, e a quem tributamos o mérito e o éxito do componente de pesquisa em saúde do Programa.

\section{SUMMARY}

\section{Coordination, assessment and follow-up procedures of health research projects of Polonoroeste}

The activities of coordination, following-up and assessment of the Integrated Development Program of the Northwestern Region of Brazil - POLONOROESTE - are analised, based on the general and specific objectives and goals of the program, and on partial results achieved.

Special emphasis was given on the technical appraisal of the research projects, under supervision of the Coordination of the Health Sciences of CNPq - (National Council for Science and Technology Development). Following the year of 1986 , it was decided to go deeper on the auditing activities, as well as assistance, follow-up in a continuous way, and training of local professional in more developed centers.

Since Science and Technology have had an increasingly pervasive influence on almost every aspect of human affairs, the results of the research projects are likely to have major beneficial effects on the society, and, in this particular case, among those communities linked to the "POLONOROESTE".

After five years, the results of this investment provide information regarding our position in this Project, what investigations and approaches appear most likely to yield further investigation, and what we might do, to reach a state of better planning and management strategies.

Presenting such a set of perspectives and assembling the views and results of some of the foremost researchers of the present health research project, their results are showed out and will give support to government members in planning, execution, and evaluating the effectiveness and eficacy of responses to the community needs.

The task of preparing this special issue belongs to the researchers involved with the "POLONOROESTE", - and to the Editorial review of Dr. Mario Montenegro and Dr. José Brandão Neto. It has certainly been extremely grateful to CNPq police-makers to have had the opportunity of managing this program and apreciating the relevance of the results presently published.

\section{REFHREACIAS BIBIIOGIRAFICAS}

I. BANCO MUNDIAL. Documento N: 35376 - Relatório de avaliacão do quadro de pessoal, primeira fase do projeto saúde do Programa de Desenvolvimento Integrado da Região Noroeste do Brasil. Washington, EUA, 1981

2. BANCO MUNDIAL. Relatório da missào de preparacâo do Projeto de Desenvolvimento Rural em Rondônia, FAO Programa de Cooperativas do Banco Mundial. Washington, EUA, 21 de novembro de 1980.

3. BELINATI, W. - Avaliação de meio-termo do compo nente de pesquisa em saúde do Programa de Desenvolvimento Integrado do Noroeste do Brasil. Brasilia, CNPq, 1985.

4. MINISTERIO DA SAUDERSUCAM - SUPERINTENDENCIA DE CAMPANHAS DE SAÚDE PÜBLICA. Combate à malária cm Rondônia, período 1980 a 1986. Brasilia, 1987.

5. MINISTEERIO DO INTERIOR, MINISTERIO DA AGRICULTURA, MINISTERIO DOS TRANSPORTES. Programa Integrado de Desenvolvimento do Noroeste do Brasil. Área de influência da ligaçào rodoviária Cuiabá-Porto Velho. Brasilia, 1979.

6. MINISTERIO DO INTERIOR/SUDECOSUPERINTENDENCIA DE DESENVOLVIMENTO DO CENTRO-OESTL: Acordo de empréstimo para o 
BELINATI, W. \& COSTA E SILVA, A. - Coordenação, avaliação e acompanhamento dos projetos de pesquisa em saúde do Programa Polonoroeste. Rev. Inst. Med. trop. São Paulo, 30(3):125-136, 1988.

Programa de Desenvolvimento da Regiào Noroeste Primeira Fase: Projeto de Saúde - entre a República Federativa do Brasil e o Banco Internacional para a Re construção e Desenvolvimento. Brasília, 15 de dezembro de 1981.
7. MINISTERIO DO INTERIOR/SUDECO - SUPERINTENDÊNCIA DO DESENVOLVIMENTO DA REGIĀO CENTRO-OESTE. Relatórios de monitoria do Programa Integrado de Desenvolvimento da Região Noroeste do Brasil. Brasília, 1986 e 1987. 